\title{
Design of a Simplified-theory, Hands-on Microcontroller-based Design Lab for Use by First Year Students
}

\author{
T.C. Muench, P.Eng., \\ Associate Professor of Engineering \\ Listwin Family Chair in Innovative Teaching \\ University of Saskatchewan \\ tim.muench@usask.ca
}

\begin{abstract}
The design portion of a second term freshman course, 'Introduction to Engineering II', provides an overview of each of the disciplines within the College, and a representative, discipline-specific design lab.

With only 2 hours of pre-lab lecture/preparation, and 3 hours in-lab time, providing the necessary theory and completing a meaningful lab is extremely challenging when the fundamental discipline-specific knowledge is still very limited.

This Electrical Engineering lab requires students to modify a program to implement a microcontrollerbased combination lock. It simulates a hotel room door lock, except that it uses a key pad instead of a swiped card.

This paper investigates the design of the lab, its implementation, and feedback results.

\section{Introduction}

Selecting an engineering discipline is likely the most important decision an engineering student will make with respect to their career. It is very important to provide students with enough information and experience to make such a decision. The design portion of this 'Introduction to Engineering II' course provides an overview of each of the disciplines within the College, and a representative, discipline-specific design lab. With a first-year, second term course, the challenge is creating a meaningful design problem when the fundamental discipline-specific knowledge is very limited.
\end{abstract}

\subsection{Lab Overview}

This paper looks at the development of an Electrical Engineering lab. With only 2 hours of pre-lab lecture/preparation, and 3 hours in-lab time, providing the necessary theory and completing a meaningful lab is extremely challenging.
The designed lab requires students to design and implement a microcontroller-based combination lock. The frame of reference is the locks used on hotel room doors, except with a manually keyed combination in place of a swiped card. In reality these systems are battery powered, and are not electrically connected to anything, so they use a pseudo-random sequence of codes to recognize the current code, and the next one that will be generated by the front desk (using the same pseudo-random generator).

A number of options were considered for the implementation, with various degrees of user control and instruction set complexity.

Students are provided with a working copy of a single combination program, which simulates a keyless entry for a garage door opener. Much of the difficult keypad scanning and combination display operations have been done, so students are only required to create a few relatively straightforward subroutines, declare some variables, and make some changes to the flow and logic of the main program.

This paper discusses the iterative process of designing and testing of the lab, and the development of related lecture material and lab overview. Also discussed is the lab implementation, as well as the results of student feedback as to its difficulty and effectiveness in accomplishing the goals of the design.

\subsection{Overview of Paper}

Section 1 provides an overview of the general lab concept and an overview of the paper.

The selection of lab topic, the acquisition of the hardware and the development of the required code are discussed in Section 2.

Section 3 details the preparatory background material and the lab overview that were provided to students during lecture sessions

Lab implementation and logistics are discussed in section 4.

Section 5 investigates the results of student surveys and the information gleaned from feedback/comments. 
Finally, Section 6 deals with the conclusions that were drawn from the lab implementation and feedback.

\section{Lab Selection and Development}

The proposed lab must be representative of the Electrical and the proposed Computer Engineering disciplines. It must also be developed with the understanding that the students do not have any related background, and that there is only limited time to prepare them with the necessary background theory.

\subsection{Selection of Lab}

Discussion of various options and their feasibility were held between the representative from the Department of Electrical Engineering, Dr. Eric Salt, Professor of Engineering, and the author. It was determined that the implementation of a hotel room electric door lock would be suitable.

\subsection{Lab Development and Lab Handout}

A single board microprocessor system by Motorola was selected to accommodate the lab design and, because of the promotion aspect of electrical engineering, the Department of Electrical Engineering agreed to supply the boards, and the faculty resources to develop the lab.

The original plan was to create a monitor program and a reduced instruction set that would be interpreted by the monitor to create the lock program. It became obvious that this would take a great deal of work, and the solution would be good for only a single application, and would require a lot of maintenance if the lab were to be modified.

The new approach was to develop a reusable core of input/output routines, and then a main program specific to the lab, which could be modified by the students, In the future, a new lab could be created by re-using the core routines, and creating a new main program. More than 2 weeks full-time effort was required to get the lab and lab handout [1] to a workable condition, but it will now be viable to come up with new labs in the future, with a much reduced time investment.

\subsubsection{Description - Motorola Microprocessor}

The Motorola microprocessor board integrates the microprocessor, a hexadecimal keypad, a 4 digit 7segment display, as well as a number of other banks of inputs/outputs which were not used for this lab.

Assembler software was also included, as well as debugging software. Due to the short time period, and the complexity of the debugging software, formal debugging of the executable processor code was not implemented in the lab.

\subsubsection{Lab Details}

Section 3.2 of this paper goes into detail with respect to the lab overview given to the students. The discussion of the lab contents has thus been deferred to that section.

\subsection{Lab Testing}

Following the development of the lab and the lab handout by Professor Salt of Electrical Engineering, the critical step of testing the lab and reviewing the handout was performed by the author.

It can not be overemphasized how critical it is to ensure that the lab handout and the lab process are fine tuned to ensure smooth operation in the lab setting. It can mean the difference between success and disaster!

The lab was checked for clarity, accuracy, omissions, sufficient background.

\subsubsection{Addition of debugging code}

It became obvious that once code was downloaded to the processor and run, there was no effective way to debug the code because learning the debugging tool was out of the question.

A block of debugging code was written, which could be pasted into the student's program. By typing in special combinations (all 8's or all 9's), the combination and alternate combination, which are the two most important bits of information to debug this lab, can be temporarily displayed to the output display.

The debugging code was made available for both instructors and students that wanted to display the combinations for debugging purposes.

\subsection{Lab Presentation Format}

Due to the 20 page length of the original lab handout, it was decided that 2 options would be made available to the students. Part of the student survey conducted after the lab asked which of these two formats was more desirable. Results are shown later in this paper.

\subsubsection{Initial format}

The initial format of the lab had the background and theory for the microprocessor and the hotel room door lock embedded in the lab procedure handout. As mentioned, the combined document was 20 pages in length, which raised some concern about whether or not students could make it through a 20 page document in a 3 hour lab period.

\subsubsection{Alternate format}

An alternate format for the lab was edited to provide a separate theory portion - a Powerpoint presentation that was used in the lab overview lecture, and a 
condensed lab handout, essentially containing only the directly related lab instructions. This cut the lab down to a more manageable 9 pages.

\subsection{Resources Required}

A number of resources were required to implement this lab, including human resources, hardware, and computer lab facilities. These resources are discussed at further length in related sections that follow.

\section{Background Information for Students}

To make the lab meaningful to the students, they must have an understanding of the key principles, and an appreciation for some of the related peripheral concepts.

Two sessions were available for providing students with the background required for the lab. As part of a new experiment to provide extra background for labs, a 1.5 hour lecture provided general background theory on binary/hex numbers, logic and microprocessors. A 30 minute lab overview provided details specific to the lab, and any necessary pre-lab preparation.

\subsection{General Background Theory Lecture}

The 1.5 hour lecture to provide background theory, entitled 'Introduction to Digital Computing' was developed by Dr. Hugh Wood [2]. Dr. Wood is the former Head of Electrical Engineering, and currently is Chief Operating Officer for VCOM, a leading designer and manufacturer of products for the Cable Television (CATV), Data over Cable, Digital Video, and Wireless Telecommunications industries.

Dr. Wood also makes room in his busy schedule to return annually as a Sessional Lecturer for the Introduction to Engineering I and II courses.

The presentation was posted on the class website for the students to access for further review, if necessary.

The following sub-sections summarize the information that was provided in the background theory lecture.

\subsubsection{Background}

The presentation discusses how digital computing is pervasive in all areas of engineering, and therefore, fundamental understanding of it is necessary. At its lowest level, computer hardware is reduced to inputs, outputs and logic.

\subsubsection{Logic}

To provide an understanding of Logic, some examples were discussed to reinforce the concept of Logic. The examples were of button pressing on a calculator, and process control on a bottling line, which were both reduced to inputs, outputs and logic operations.

\subsubsection{Flow Charts}

Flow charts are used in the lab. To provide some basic understanding, a flow chart of the calculator button pressing implementation was provided.

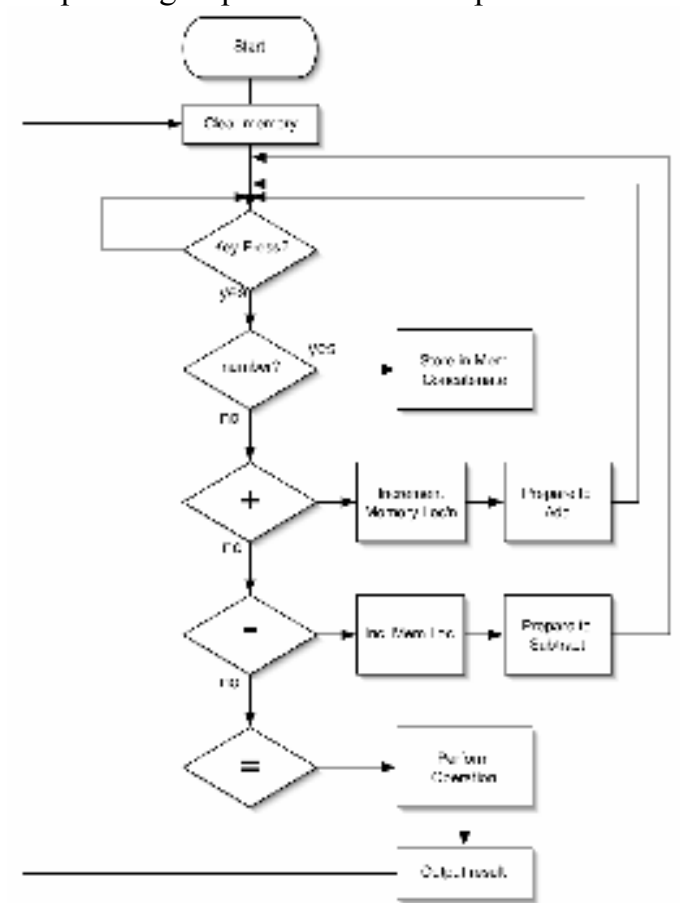

Figure 1 - Sample flow chart presented

\subsubsection{Number Systems}

One of the steps in the lab uses a binary filter, and another uses hex numbers, so it is important that the students understand other number systems. Students learned the fundamentals of the binary number system, based on the two states (ON/OFF or $0 / 1)$ that are available with computer system hardware/logic. Number systems were discussed, starting with base 10 , then binary, then hexadecimal, and examples show the calculation and conversion of numbers.

\subsubsection{Arithmetic}

Decimal and hexadecimal examples of how addition is done were provided so that students could see that the addition operations are the same, except the base of the numbers differs.

\subsubsection{Binary Arithmetic}

The addition of binary numbers was considered separately because of the link to microprocessors, and the ability to implement the addition operation using logic and hardware. 
The example provided shows that a binary addition is an 'Exclusive Or' logic operation, with the carry being considered just another digit.

\subsubsection{Field Effect Transistor (FET) / Logic Gates}

Logic operations, which are the building blocks of computer hardware, require switching operations. The use of Field Effect Transistors is a method for implementing the switching with a solid state device.

An example circuit provided in the lecture shows how FETs and resistors can be combined to implement a NAND gate.

Examples of the basic logic gate symbols were shown, as well as how primitive gates can be combined into more complicated ones such as XOR, as shown in Figure 2.

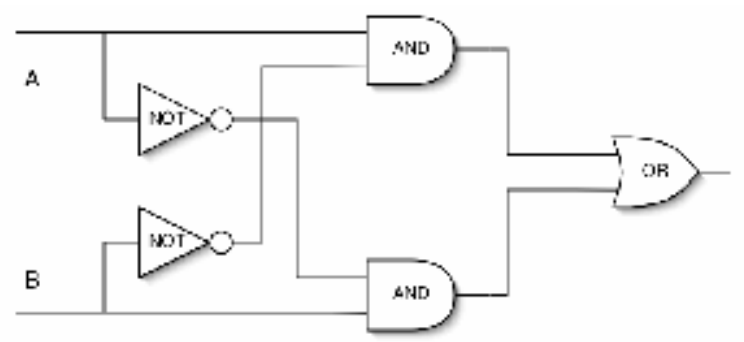

Figure 2 - XOR gate created from primitive gates

\subsubsection{Logic Circuits}

The overview showed examples of how many other circuits are built from the basic logic gates. The discussion included:

Adder

Memory - dynamic - used because it's cheap

Flip-flops - a form of static memory

Latches - clocked flip-flops

Registers - made from several latches

Shift Register - used for serial communication (network data transmission), or if configured to transfer out simultaneously, a data bus.

\subsubsection{Arranging Components into a Processor}

At the point where the components and building blocks have been described, the students were introduced to combination of registers, a bus, an adder and other components to create a processor. A basic simplified block diagram is shown in Figure 3. By bringing all of the components together into a processor it shows how the concept of a processor is an extension of the previous concept where simpler components are combined into more complex devices.

\subsubsection{Instructions}

The lab uses basic assembly language instructions for programming the microprocessor, so the students have to gain an appreciation for how instructions operate at a low level.

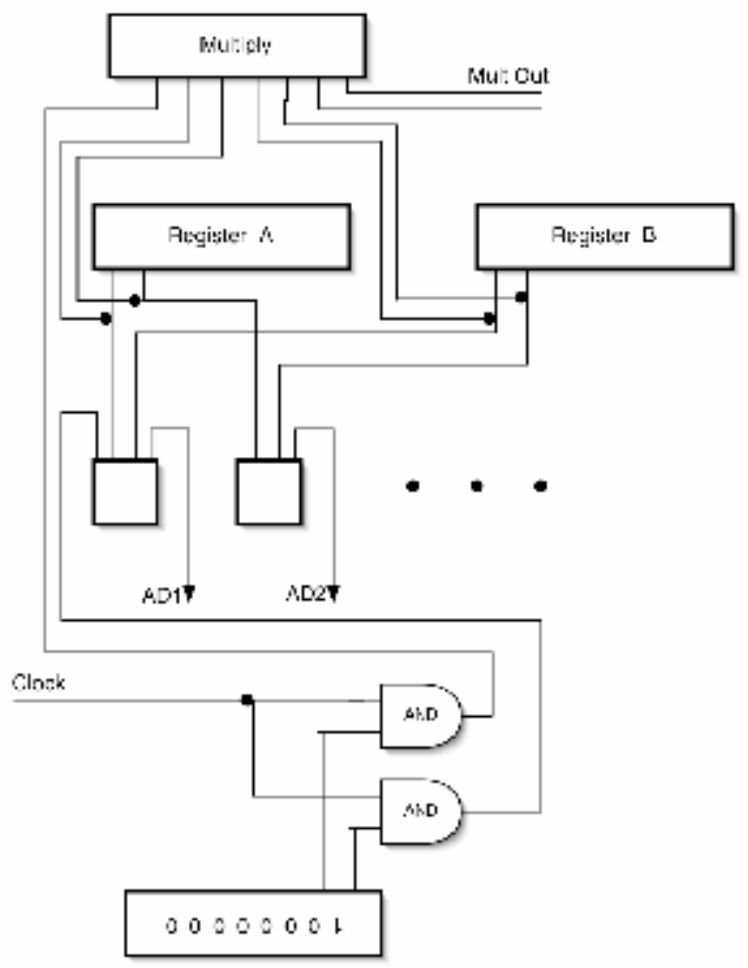

Figure 3 - Microprocessor block diagram

The concept is presented that Load, Add, and other commands are also represented by binary numbers, but are stored in program memory as opposed to data memory. The concept is also presented that data is loaded into registers, operations are performed in the registers, and result is either retained in the register, or sent out to data memory.

\subsubsection{Input and Output}

Basic input and output was discussed, with special attention to keypad entry and 7-segment displays, both of which are used in the lab. Also discussed was the fact that all inputs and outputs are also represented by binary codes.

\subsubsection{Compiler}

The last piece in the puzzle for the lab was the explanation of the concept of a compiler. The fact that humans prefer to see meaningful symbols rather than binary codes to represent commands leads to the introduction of representations such as LDAA LoaD Accumulator A) are translated into the corresponding code by the compiler.

Others commands such as ADDA (ADD to accumulator A), TST (to see if a result is zero) are 
used to represent the equivalent binary instruction, and the compiler converts them to their binary equivalents when it's time to make executable code.

\subsection{Lab Overview Lecture}

The lab overview lecture was created by extracting the basic theory portion from the original lab handout, and editing/adding to it to create a structured presentation to prepare the students directly for the lab.

The Powerpoint presentation developed for the lecture was also posted to the class website so that students could review it if necessary.

\subsubsection{Overview of Microprocessor Board}

The introduction of the lecture spoke first to the fact that microprocessors are found not only in computers, but in a wide array of applications such as Cable TV modems, engine ignition/fuel control, microwave ovens etc.

Since students are required to set up and connect the microprocessor as part of the lab, the locations of important items such as the reset button, the keypad and display, and the power and serial connections were all illustrated.

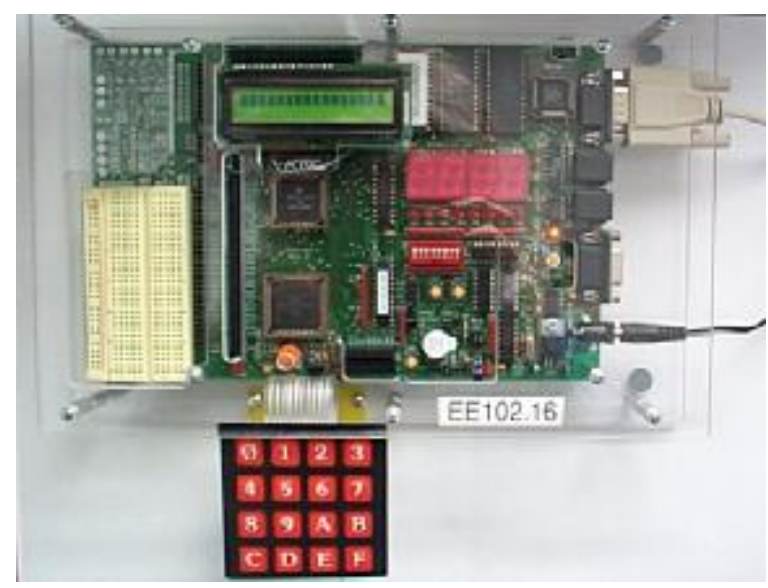

Figure 4 - Microprocessor board

\subsubsection{Steps to Programming the Microprocessor}

Following the correct procedure is required to allow students to execute changes to their code. The following procedure, and the significance of each step, was discussed to ensure students were familiar with it prior to the lab, and that faculty or Lab TAs would not have to explain it repeatedly in the lab.

1. Write/Edit source code with text editor (or assembler program)

2. Connect the microprocessor to the PC with serial cable
3. Run an assembler to produce binary code (instructions) for the microprocessor

4. Reset micro board to run monitor program

5. Download the executable code (.s19 file) to the microprocessor board

6. Command the monitor to run the downloaded executable program

Even with the emphasis on the steps, a number of lab problems were traced back to students missing one of the steps in the process.

\subsubsection{Downloading and Running a Program}

Students were shown the process of hooking up the microprocessor board to a PC, running the assembly/loading software, and assembling the executable code

They also observed the commands required to download the executable code to the processor, and to execute it once it has been successfully downloaded.

\subsubsection{Overview - Comments/Labels/Instructions}

The specific syntax (format) of comment statements, labels (to identify subroutines and locations to jump to) and program instructions was covered. Syntax errors are by far the most common errors that students make in the lab, and one of the most frustrating ones.

Reinforcing the importance of syntax, and the required format is aimed at reducing the chance that students will get sidetracked by a syntax error.

\subsubsection{Typical Programming Errors and Messages}

Students were given advance notice that at the start of the lab, a number of errors are intentionally introduced so that students observe the system response, and they can identify when they make some of the typical syntax errors. This will prepare them for future troubleshooting that may be required.

As part of the process, the importance of observing the line number returned in an error message was noted. Once the line number is located, a search of the typical syntax errors can be done, to put the initial troubleshooting onus on the student, rather than on the faculty / TAs. This frees the faculty / TAs up to focus on more troublesome logic or procedural problems.

\subsubsection{Description of Keyless Door Opener}

An overview of the function of the keyless door opener program was given. This program was the initial starting point for the students, and modifications to it are required to create a hotel room door lock.

The description indicates that as keys are pressed on the keypad, the value of the key that has been pressed is stored in the lowest digit of the combination buffer. 
All other entries are shifted over one digit. Once 4 values are stored in the 4 digit code, the newest digit shifts in, all others are shifted over, and the oldest entry is discarded. At any point, if the combination in the buffer matches the set combination, the open door command is issued, and the word OPEn is displayed on the 4 digit, 7-segment display (to represent the activation of a door opener).

A number of components such as initialization, scanning the keypad, refreshing the output display, shifting numbers in the combination register, and executing the 'Open Door' command were discussed, but their detailed function was not required to be understood.

It was explained that the subroutine that defines the combination must be copied and modified to create an alternate combination. The subroutine that checks the combination will also be copied and modified to check for the second combination.

\subsubsection{Modifying a Hard-Coded Combination}

Students were shown how to manually edit the initial hard-coded combination as the first simple programming test.

\subsubsection{Writing a Routine to Change Combination}

The next programming step that was introduced was to create a new subroutine called change combo, which uses a predefined algorithm (in this case simply adding 4 to each digit) to change the combination.

It was explained that to test this routine, the main program must be temporarily modified to call this subroutine every time the door is successfully opened. This causes a rolling combination that is changed every time the subroutine is run.

\subsubsection{Creating an Alternate Combination}

A description was provided with respect to the next modification to the program, accepting a second combination. This involved first defining the memory to hold the second combination and then initializing it. Next a copy of the check_combo subroutine has to be modified to check the alternate combination. Finally, the Main program has to be modified to check both.

The program logic to implement the change to the Main program is slightly complicated for those that are not familiar with assembly language, so it was STRONGLY suggested that they pay close attention to this part of the presentation if they wanted to save themselves time and anguish in the lab.

The function of the existing main program was explained (only about 16 lines, 3 of which were labels) and the 3 key steps involved in the combination test were explained in detail. The logic involved with making sure both combinations were tested was explained, and a solution was alluded to. It involved a second set of the 3 key steps used to check the main combination, and two labels to facilitate the required branching operations. Essentially the horses were led to the water, without actually making them drink.

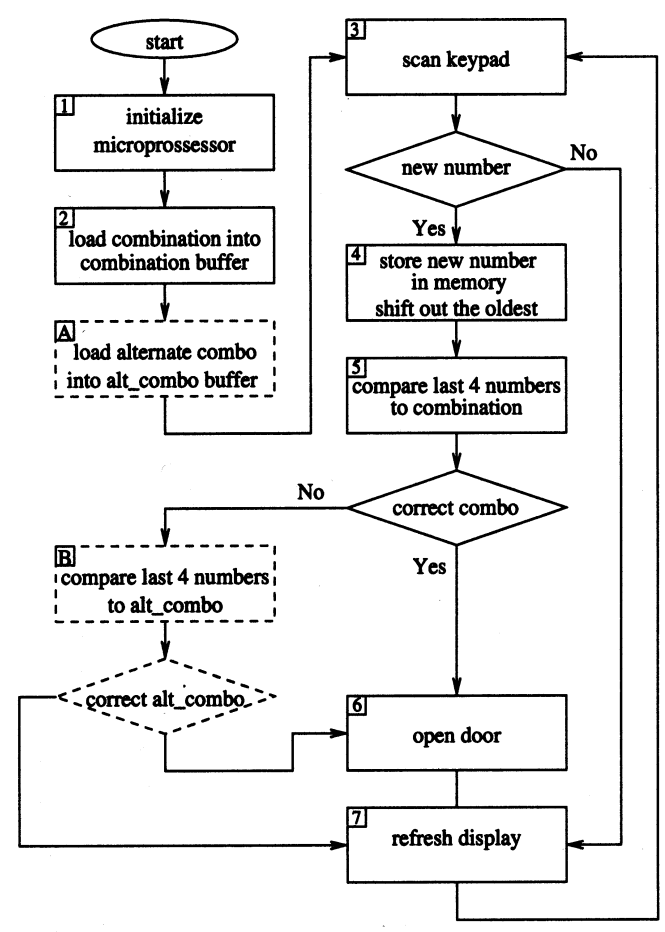

Figure 5 - Flow Chart - Alternate Combo

\subsubsection{Description of a Hotel Room Lock}

A description of the operation of a hotel door room lock was provided. These locks are battery powered and have no electronic connection to the computer at the front desk. Real locks use magnetic strips, but the lab uses a keyed-in combination instead.

The hotel door lock recognizes 2 combinations. One is called the current-occupant combination, and the other is called the next-occupant combination. The next-occupant combination is related to the currentoccupant combination by an algorithm. In reality, this would be a complicated algorithm. Our lab will simply add 4 to the current-occupant combination to create the next-occupant combination.

When a new guest checks into the hotel the clerk gives the new guest the next-occupant combination. This is possible because the clerk's PC (the computer at the front desk) keeps track of the current-occupant current-occupant combination for each room, so it can calculate the next-occupant combination using the same algorithm as the locks. 
When the new guest opens the door with the nextoccupant combination for the first time, the lock changes both combinations. The current-occupant combination is changed to that of the next-occupant combination and a new next-occupant combination is created by the algorithm.

The combination used by the guest that checked out will not work once the next guest has opened the door (with the next-occupant combination). This also means the new guest becomes the current occupant upon first entry into the room.

In a real system, other information, such as scheduled checkout time, and combinations for housekeeping staff, may also be contained on the magnetic key. The scheduled checkout time can be used to disable the key (current-occupant combination) even if the next-occupant has not used their key yet.

\subsubsection{Designing/Implementing the Hotel Lock}

Instructions were discussed that the students were to start with the two-combination program that they will have just created. A modification is required that will changes both combinations when the alternate combination (the next occupant combination) is used to open the door.

There are a number of different ways that this can be accomplished, some more difficult than others. About the simplest way to construct both of the new combinations is by running the 'new combination' algorithm (adding 4 to each digit) on BOTH of the old combinations.

Although this may not be directly obvious, functionally it is the same as copying the nextoccupant combination to the current-occupant combination, and then generating a new next-occupant combination from the newly generated currentoccupant combination.

\section{Lab Implementation and Logistics}

Physically accommodating nearly 400 students in a hands-on microprocessor lab is a challenge.

\subsection{Lab Resources and Scheduling}

Due to start-up costs, and a maximum daily lab size of 19 groups of 4 students, it was decided to purchase only 24 microprocessor boards. This allowed the accommodation of the largest lab session, and provided a buffer in the event of a failed microprocessor board.

The current lab schedule for Introduction to Engineering II has access to a 45 station computer lab for five 3-hour lab periods per week. As mentioned, the largest of the 5 lab sessions has 19 groups, each of which has 4 members. Fortunately, the software and computing power required were both minimal, so a second suitable lab of 32 stations was available, and it was also booked. In total, this allowed seating for exactly 19 groups of 4 . Although only 1 computer was required per group, physical seating of all group members required the larger lab size.

\subsection{Faculty/Teaching Assistants}

Each lab session in the course has 1 faculty member and 1 Teaching Assistant assigned, so one of them was assigned to cover each computer lab. In the larger of the 2 labs, this meant that the worst case was 1 person in a lab with 11 groups.

To minimize the chances of problems, and to get all groups up and operating, the Start-up was done as a group, and students were guided through the connection and setup of the microprocessors.

In addition, the workload on the lab personnel was minimized through the reliance on a well planned and well prepared lab handout. In general lab groups only had waits of a few minutes before instructors came available to answer questions.

\subsection{Specification of Deliverables}

The deliverables in the Lab report required a technical report format, referring specifically to the design exercise, which was the development of the hotel room lock. Discussion, in general terms of the modification and creation of code to implement the new design, and discussion of some of the design options by which the rolling codes could be implemented (design alternatives). A complete copy of the working code was required to be included in an appendix.

\section{Student Feedback}

A student survey was conducted to determine their reactions to the lab and their opinions on how it affected their design skills.

\subsection{Student Survey Feedback}

A voluntary survey was conducted to acquire student feedback on the lab. Part 1 was designed as a categorical response to indicate the degree of agreement with statements related to the lab, or the students perception of the lab. The response options were:
1. Strongly Agree
2. Agree
4. Somewhat Disagree
3. Somewhat Agree
5. Disagree
6. Strongly Disagree

\subsubsection{Sample Size}

Although participation was voluntary, 221 surveys were returned, which is significantly over half of all the registered students 


\subsubsection{Handling of Incomplete Records}

Question were independent, so responses were still used when a student's overall record was incomplete.

\subsubsection{Results}

For each of the questions that follow in Figures 611, a detailed distribution of student responses is shown, followed by a Total of Agreement responses versus Total of Disagreement responses.

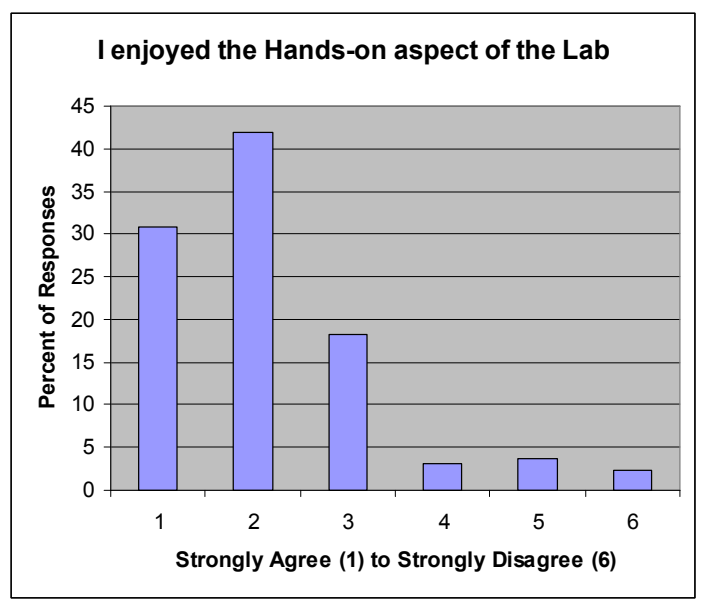

Figure 6 - Enjoyed hands-on aspect of the lab Agreement - 91\% Disagreement - 9\%

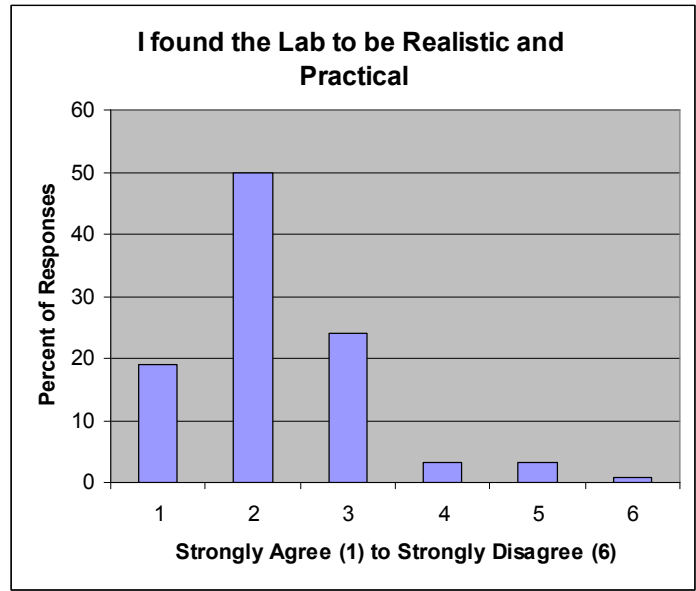

Figure 7 - Lab was realistic and practical Agreement - 93\%

Disagreement - 7\%

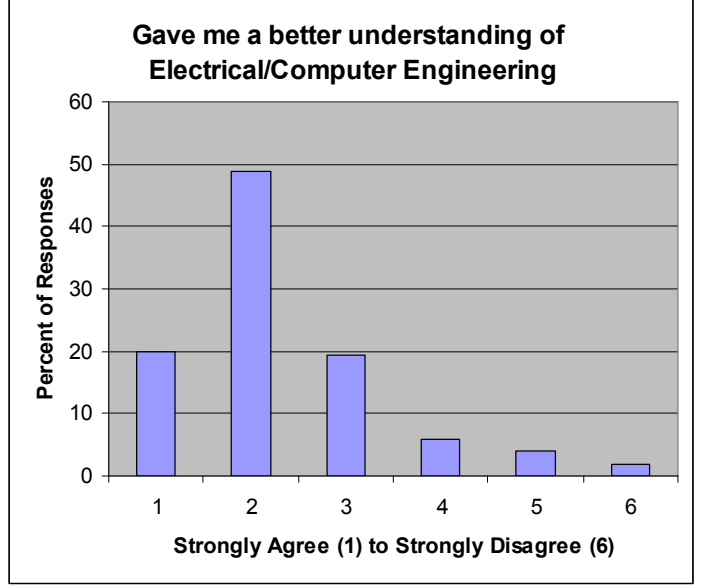

Figure 8 - Better understanding of Electrical Eng'g Agreement - 88\%

Disagreement - 12\%

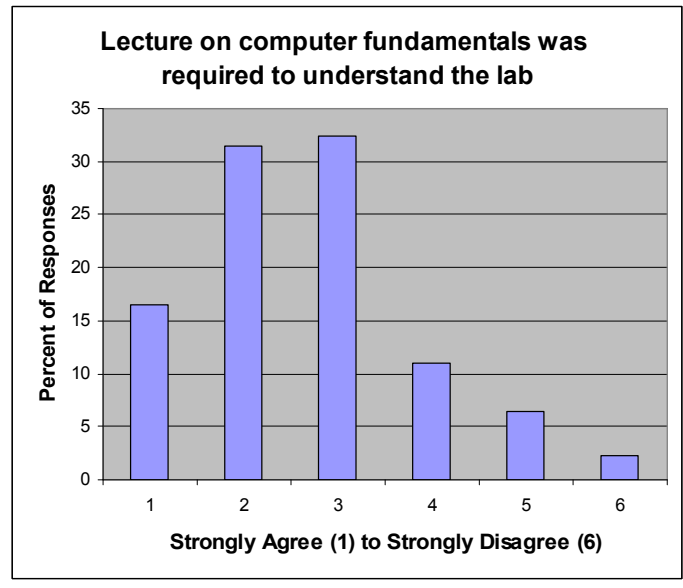

Figure 9 - Computer fundamentals lecture req'd Agreement - 80\%

Disagreement - 20\%

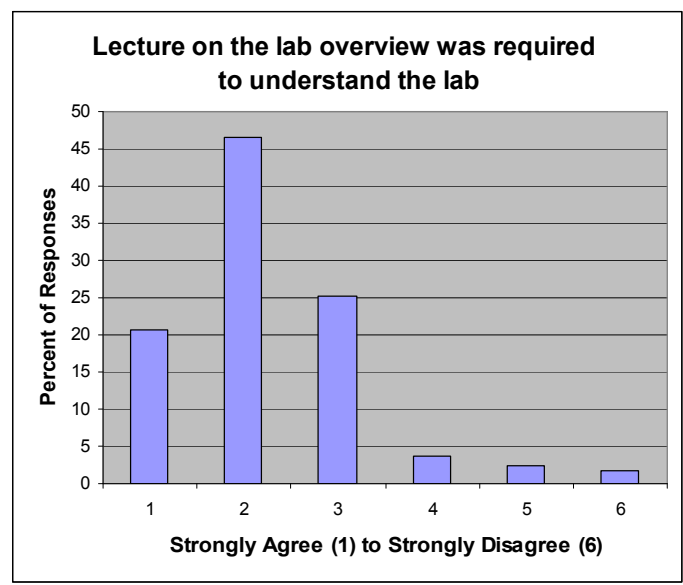

Figure 10 - Lab overview lecture required Agreement - 92\%

Disagreement - 8\% 


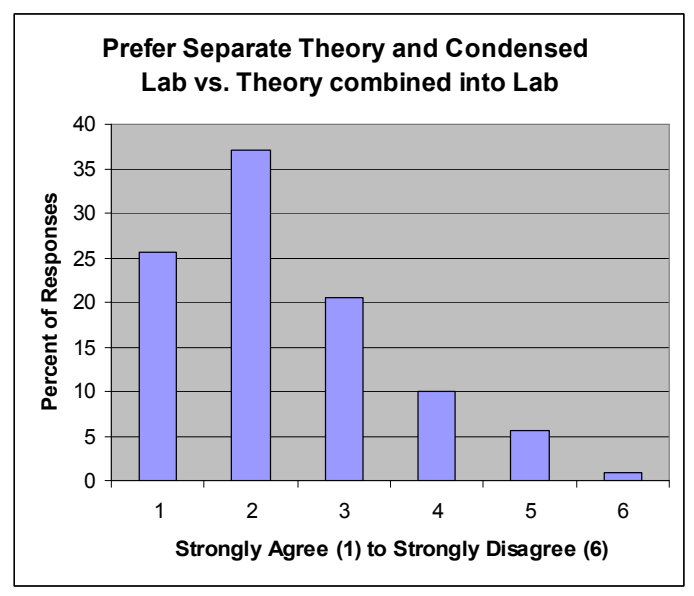

Figure 11 - Prefer separate theory \& lab handouts

Agreement - 83\%

Disagreement - 17\%

\subsubsection{Summary of Results}

The responses to the four questions listed below were overwhelmingly in agreement, with approximately $90 \%$ of the responses in agreement, or 9 of 10 respondents.

I enjoyed the hands-on aspect of the lab

I found the lab to be realistic and practical

The lab gave me a better understanding of Electrical/Computer Engineering

The lecture on the Lab Overview was required to understand the lab

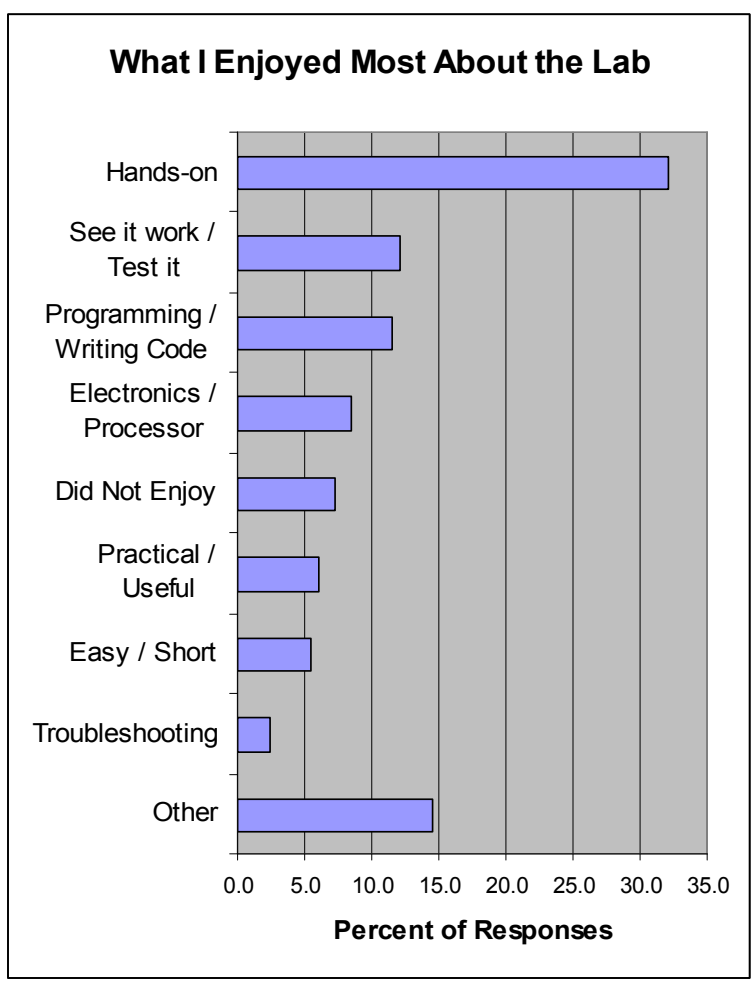

Figure 12 - Enjoyed Most About Lab
The remaining two questions that follow were still very strongly in agreement, at approximately $80 \%$, or 4 of 5 respondents.

The lecture on Computer Fundamentals was required to understand the lab

I prefer separate theory and a condensed lab handout versus having theory combined into the $l a b$

\subsection{Student Comments}

Two questions were designed to elicit responses from students, to allow more freedom of response.

The two questions were:

What I enjoyed most about the lab

What I found most difficult about the lab

The number of written responses to the questions were 165 and 136 respectively. Responses were examined for common themes and were then categorized according to the common themes. The results are shown in Figures 12 and 13 respectively.

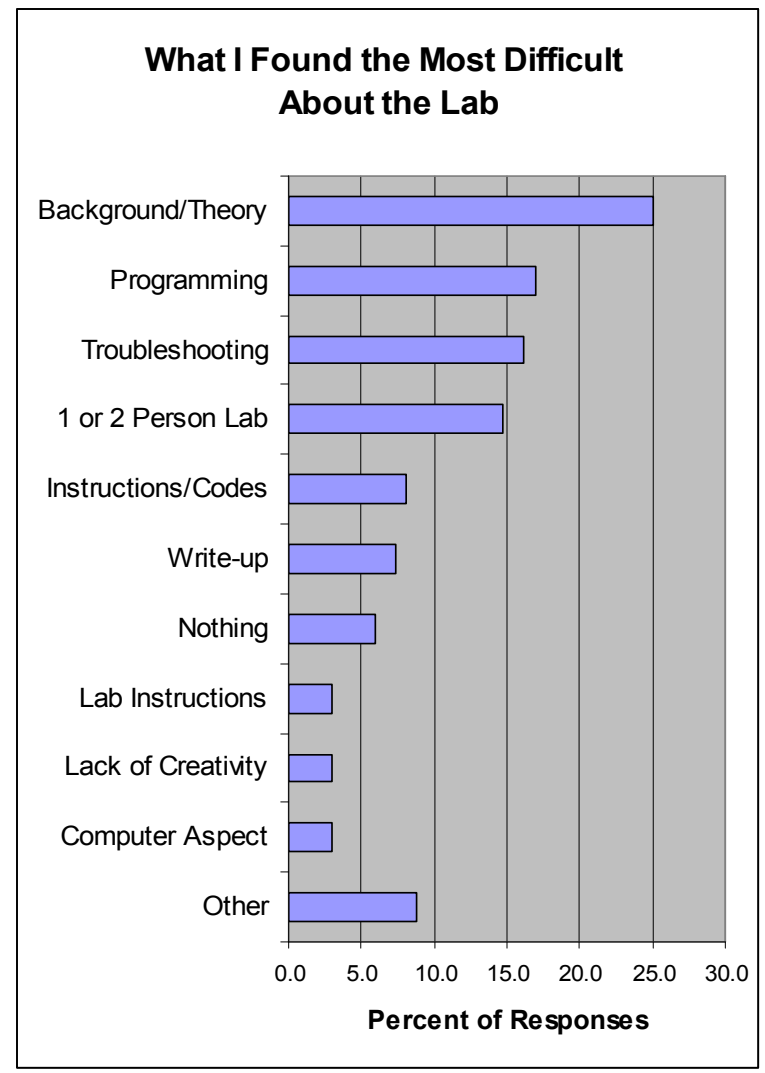

Figure 13 - Found Most Difficult About Lab

\subsubsection{General Trends and Recurring Themes}

The major enjoyable aspects of the lab are summarized by hands-on and testing, followed by 
programming. All of these are characteristics of a hands-on lab.

By far, the majority of the comments on the 'most difficult' portion of the lab were related to the lack of related background/theory and then programming/ troubleshooting, as would be expected in a lab such as this.

It is interesting to note that there were only a few percentage points difference between those who found programming difficult, and those who found it enjoyable!

\subsubsection{Interesting Comments}

Some responses to the 'most enjoyable part of the $l a b$ '

'The "oooooh it works" factor (I did that factor)'

'That I had never done anything like it, programming something and watching the results'

\section{Conclusions}

The objective of this work was to determine if it is possible to have a successful hands-on microprocessor-based design lab for first-year students.

Based on the observed results and student feedback, the attempt was indeed successful, as is elaborated in the following conclusions.

\subsection{Lab Development}

The lab was developed with the intent that it provide an introduction to Electrical and Computer Engineering, while not assuming any prior knowledge in the area.

The lab was planned in a way such that the basic program can be reused in the future by coming up with a new device that uses the same basic input/output modules, but with a new main program. The 'Introduction to Digital Computing' lecture is also generic enough to be reusable.

Ensuring that the lab handout was complete and checked for errors and missing information was absolutely critical to the success of the lab.

\subsection{Background Preparation Success}

Another critical key to the success of the lab was proper background preparation. Having the students properly prepared resulted in a shortest completion time of a half hour. All but 5 of the approximately 100 groups finished within the allotted 3 hour lab period. The remaining 5 groups had to sign out boards to complete the lab at a later time.

\subsection{Student Feedback}

Student feedback was overwhelmingly positive. Questions related to: Hands-on aspects, realistic and practical aspects, a better understanding of Electrical and Computer Engineering, and the need for the lab overview lecture that was provided,were all supported by agreement of 9 out of 10 students.

Questions related to: The need for the lecture on computer fundamentals that was provided, and the preference for a separate theory and condensed lab handout, as was provided, were all supported by agreement of 4 out of 5 students.

Student comments on the most enjoyable part of the lab focused on the hands-on aspect, and the ability to see results. Comments on the most difficult part of the lab were summed up by the lack of related background. A certain degree of this was expected with the lack of electrical background of the students, however, it was noted that during the pre-lab lecture, numerous students were observed not paying attention. This lack of attention may have been due to the fact that students who were not lab manager for this lab (manager writes the report), and who may have already done their semester's duty as lab manager, may have thought that they could slide through without paying attention.

\subsection{Summary}

With the exception of a lack of background expressed by some students, which may be explained by inattention, the vast majority of the students competed on time, and provided overwhelmingly positive feedback about the lab.

\section{References}

[1] JE. Salt, "GE 120 Laboratory for Electrical Engineering", Unpublished laboratory handout GE120, University of Saskatchewan, 2005.

[2] HC. Wood, "Introduction to Digital Computing", Unpublished lecture notes - GE120, University of Saskatchewan, 2005. 